\title{
Articlelnsulin resistance and cardiometabolic indexes: a comparison of gender-related differences in working-age subjects with overweight and obesity
}

\author{
Luisella Vigna \\ Ospedale Maggiore di Milano Policlinico: Fondazione IRCCS Ca' Granda Ospedale Maggiore Policlinico \\ Amedea Silvia Tirelli \\ Ospedale Maggiore di Milano Policlinico: Fondazione IRCCS Ca' Granda Ospedale Maggiore Policlinico \\ Melania Gaggini \\ Istituto di Fisiologia Clinica Consiglio Nazionale delle Ricerche \\ Salvina Di Piazza \\ Ospedale Maggiore di Milano Policlinico: Fondazione IRCCS Ca' Granda Ospedale Maggiore Policlinico \\ Laura Tomaino \\ Polytechnic University of Marche: Universita Politecnica delle Marche \\ Stefano Turolo \\ La Fondazione IRCCS Ospedale Maggiore Policlinico: Fondazione IRCCS Ca' Granda Ospedale Maggiore Policlinico \\ Gianluca Moroncini \\ Azienda Ospedaliera Ospedali Riuniti Marche Nord \\ Kyriazoula Chatzianagnostou \\ CNR di Pisa: Consiglio Nazionale delle Ricerche Area della Ricerca di Pisa \\ Fabrizia Bamonti \\ Università degli Studi di Milano: Universita degli Studi di Milano \\ Cristina Vassalle ( $\sim$ cristina.vassalle@ftgm.it) \\ CNR di Pisa: Consiglio Nazionale delle Ricerche Area della Ricerca di Pisa https://orcid.org/0000-0003-3438-6450
}

\section{Research Article}

Keywords: occupational medicine, working-age subjects, cardiometabolic risk, cardiometabolic indexes, insulin resistance indexes

Posted Date: January 27th, 2022

DOI: https://doi.org/10.21203/rs.3.rs-1257473/v1

License: (c) (1) This work is licensed under a Creative Commons Attribution 4.0 International License. Read Full License 


\section{Abstract}

Purpose: The aim of the study was to evaluate indexes of insulin resistance and cardiometabolic risk in a large population of workers with overweight or obesity, evidencing possible gender-related differences, in order to identify a possible efficient, cheap and simple strategy to apply in clinical practice of workers surveillance. Methods: The evaluation of IR and cardiometabolic risk indexes (HOMA, QUICKI, Ty/HDLC, TyG, insuTAG Castelli risk indexes 1 and 2, non-HDLC, TRL-C, AIP, and VAI) was performed in a population of 1195 working-age subjects with overweight or obesity (322 males, mean age $49 \pm 11$ years). Results: The prevalence of all IR and cardiometabolic risk was higher among males for all indices.Aging, waist circumference, BMI, blood pressure, glucose, CRP, fibrinogen and uric acid were correlated more frequently with IR/cardiometabolic indexes in women, homocysteine in men. The percent of the workers identified as insulin resistant, IR+, or at higher cardiometabolic risk greatly vary according to the different index used.atly varied according to the different index used to measure risk. Conclusion: With a small group of biomarkers and anthropometric measures (fasting glucose and insulin, lipid profile, BMI and waist circumference) is possible to calculate a number of IR/cardiometabolic indexes, which likely mirror different pathophysiological aspects helping in a personalized evaluation of IR and cardiometabolic risk.

\section{Introduction}

Insulin resistance (IR) and cardiometabolic abnormalities affect a most of the middle-aged population of the working-age, being particularly associated with overweight and obesity[1]. Monitoring IR indexes and cardiometabolic risk is crucial in occupational medicine, because overweight/obesity is a risk factor for the development of many chronic conditions and well-being reduction, and, moreover, may have a significant long-term impact on health care costs of working-age adults[2]. Thus, it is particularly important to identify subjects at the pre-disease stage with the possibility of life-style and therapeutic intervention.

IR, due to a diminution of tissue response to insulin activity, is associated with metabolic syndrome, type 2 diabetes mellitus (T2D), obesity, and cardiovascular disease. There are several methods to assess IR state and selecting one or another depends on the purpose of their use and, often, on the available economic resources. Direct methods such as the "Hyperinsulinemic Euglycemic Glucose Clamp" or the "Insulin Suppression Test" have a low margin of error and considered the gold standard methods[3;4]. However, their application in the clinical practice or in epidemiological studies is limited as they are more complex and expensive. For these reasons, indirect methods have been developed (i.e. Insulin Sensitivity Index-ISI derived from the Oral Glucose Tolerance Test-OGTT, or Homeostasis Model Assessment-HOMAIR and Quantitative Insulin-sensitivity Check Index-QUICKI) which, even less precise, are simpler and cheaper than direct ones5-7. Nonetheless, comparison studies suggest that there could be differences in the prevalence of insulin resistance detected among different indirect indexes[8]. Recently two other indirect routine indexes based on the lipid profile and fasting glucose levels were employed: Triglycerides/HDL-cholesterol ratio (Ty/HDLC) and Triglycerides-Glucose concentrations (TyG)[9-11]. Among indirect methods, ISI, even the most expensive, better correlates with direct methods[6]. In addition, the insuTAG (fasting insulin $\times$ fasting triglycerides) has been proposed as predictor of insulin resistance (IR) and metabolic syndrome (MetS)[12].

Moreover, other indexes are currently used as indicators of cardiometabolic risk, some considering only circulating lipids (e.g. Castelli risk indexes 1 and 2 , non-HDLC, tryglicerides rich lipoporotein cholesterol-TRL-C and Atherogenic Index of Plasma-AIP), whereas others combine anthropometric measurements (such as waist circumference-WC, and body mass index-BMI) and lipid profile, as in the visceral adiposity index-VAI13-16. Accordingly, we aimed to evaluate these indexes of insulin resistance and cardiometabolic risk in a large population of workers with overweight or obesity, evidencing possible gender-related differences, in order to identify a possible efficient, cheap and simple strategy to apply in clinical practice of workers surveillance and public economic management.

\section{Materials And Methods}

\subsection{Patients}

A population of 1195 overweight or obesity workers (873 females, aged $49 \pm 11$ ) were recruited from Center of Obesity and Work, Occupational Health Unit of Clinica del Lavoro “L. Devoto”, Fondazione IRCCS Ca' Granda, Ospedale Maggiore Policlinico, Milan (Italy).

Upon entering the study, each participant signed an informed consent form and underwent medical examination. During the clinical examination, all subjects were asked to fill in a questionnaire on general health, habitual dietary intake and lifestyle (i.e. smoking history, alcohol consumption, occupation). Age, height, weight and body mass index (BMI), waist circumference (WC), systolic and diastolic blood pressure were recorded. Exclusion criteria were: history of current chronic or neoplastic disease, regular use of dietary supplements.

According to the standard recommendation of European Association for the Study of Obesity (EASO), The Obesity Society (TOS), and Obesity Canada (OC) to reduce bias and stigma associated with obesity, we used people first language[17].

The study was conducted according to the Good Clinical Practice guidelines and approved by Human Ethic Committee of Fondazione IRCCS Ca' Granda Ospedale Maggiore Policlinico (Registration number: 852).

This section may be divided by subheadings. It should provide a concise and precise description of the experimental results, their interpretation, as well as the experimental conclusions that can be drawn.

\subsection{Blood samples and laboratory biomarkers}


Blood samples were drawn after an overnight fast, to be analyzed for glucose (GI), insulin (Ins), triglycerides (Ty), total cholesterol (T-Chol), high and low density lipoprotein-cholesterol (HDLC, LDLC), gammaglutaryltransferase, creatinine, uric acid and C reactive protein (serum), and a complete blood count (CBC), fibrinogen and homocysteine levels (EDTA) by automated Modular D and P (Roche Diagnostics International Ltd, Basel, Switzerland).. Samples were frozen and stored at $-20^{\circ} \mathrm{C}$ until analysed.

\subsection{IR and cardiometabolic indexes}

- The Homeostasis Model Assessment -HOMAIR $=\mathrm{Gl}(\mathrm{mmol} / \mathrm{L}) \times \operatorname{lns}(\mathrm{mU} / \mathrm{ml}) / 22.5$

Gl=fasting glucose, Ins=fasting insulin.

Reference interval: $0.23-2.5$; IR when HOMAIR $\geq 2.5$ values.

HOMAIR index provides information about insulin effects on hepatic glucose production during the fast, but not about peripheral insulin effects during prandial phase. Log HOMAIR can be applied to exceed HOMAIR index limits $[6 ; 18]$

- Quantitative Insulin-sensitivity Check Index-QUICKI = 1/[log Ins $(\mu \mathrm{U} / \mathrm{ml}) \times \log \mathrm{GI}(\mathrm{mg} / \mathrm{dl})]$

Values fall broadly within a range between 0.45 for unusually healthy subjects and 0.30 in diabetic patients, whereas lower levels reflect greater IRs [7;19]

- insuTAG: obtained by fasting insulinxfasting triglycerides, can predict insulin resistance (IR) and metabolic syndrome (MetS) for values higher than 11[12]

- Triglycerides-Glucose, TyG:

$\operatorname{TyG}=\operatorname{Ln}[\mathrm{Ty}(\mathrm{mg} / \mathrm{dl}) \times \mathrm{Gl}(\mathrm{mg} / \mathrm{dl}) / 2]$

Ty= fasting triglycerides $\mathrm{Gl}=$ glucose, $\mathrm{IR}$ when $\mathrm{TyG}$ is $\geq 8.4[11]$.

As Ty concentrations increase during chronic hyperinsulinemia (because adipose tissue releases free fatty acids in the blood circulation) and increase in liver and heart, this index can also provide information about risk of developing cardiovascular diseases and to discriminate a prediabetes condition [20-23].

- Triglycerides/HDL cholesterol (Ty/HDL Chol): Ty/HDLC ratio is closely associated with fasting glucose levels. IR is present when this ratio is $\geq 3.5 \mathrm{mg} / \mathrm{dL}$. Ty/HDLC is a predictor for insulin resistance and metabolic syndrome, also providing information about the risk of developing the cardiovascular disease $[9 ; 10 ; 13]$.

- CASTELLI index I: This index, obtained by the total cholesterol/HDLC ratio, has close relationship with cardiovascular risk in both sexes[13].

Subjects at TC/HDL ratio greater than or equal to 4.5 were found to be at higher cardiovascular risk in the Framingham study. Optimal values are $<5$ and $<4.5$ for men and women, respectively [24]

- non-HDLC: simply calculated as the difference between TC and HDL cholesterol, represents bad cholesterol including cholesterol carried on VLDL, LDL as well as chylomicron remnants and lipoprotein(a). Several important guidelines recommended the use of non-HDLC for cardiometabolic prevention strategies and indicate this biomarker as therapeutic target[14]. A cut-off for non-HDLC values $130 \mathrm{mg} / \mathrm{dL}$ is commonly used in clinical setting[25]

- Atherogenic index-AI CASTELLI index II: This index, obtained by the LDLC/HDLC ratio, is an indicator of cardiovascular risk[13]. Ideal values are <3.5 and 3 in men and women [24].

- TLRL-C: Remnant cholesterol-TRL-C is obtained by subtracting HDL and LDL cholesterol from total cholesterol, indicating the cholesterol component of TG-rich lipoproteins (fasting very low-density lipoprotein, intermediate-density lipoprotein-IDL, and postprandial chylomicrons and their remnants). This biomarkers has been associated with inflammation and cardiometabolic risk[26]. Remnant- $\mathrm{C} \geq 30 \mathrm{mg} / \mathrm{dL}$ identifies subjects at a higher cardiovascular risk [27] 
- Atherogenic index of plasma-AIP: calculated as logarithmically transformed ratio of molar concentrations of triglycerides to HDL-cholesterol, resulting in a reliable predictor of cardiometabolic risk[14].

AIP value $<0.11$ is associated with low CVD risk 28;29

- Visceral Adiposity Index (VAI): This gender-specific index, calculate through use of simple anthropometric (BMI and WC) and laboratory (TG and HDLC) parameters, is indicative of adipose distribution and function and indirectly indicate the cardiometabolic risk [16]

\subsection{Statistical analysis}

Median, standard deviation (SD), interquartile range (IQR) and distribution curves were calculated for each index. To evaluate the degree of association between biochemical, anthropometric, and IR indexes linear regression (Pearson Correlation analysis) and two-tailed t-test. Data statistical analyses were performed with the Statview statistical package, version 5.0.1 (SAS Institute, Abacus Concept, Berkeley, CA, USA). A p value of $<0.05$ was considered statistically significant.

\section{Results}

Clinical and demographic parameters of the studied population are summarized in Table 1. There were no significant differences among males and females regarding mean age and BMI. However, levels of total cholesterol, HDL, CRP and fibrinogen were higher in women. Instead, smoking history (current-ex smokers) was more frequent in men, who presented higher blood pressure, triglycerides, fasting glucose and insulin, creatinine, homocysteine, GGT, WBC, and uric acid levels.

Table 1

Demographic, clinical and laboratory parameters in the overweight/obese

\begin{tabular}{|c|c|c|c|}
\hline & FEMALES & MALES & p value \\
\hline $\mathrm{n}$ & 1015 & 395 & - \\
\hline AGE (yrs) & $50 \pm 11$ & $49 \pm 11$ & ns \\
\hline Body mass index (kg/m2) & $34 \pm 6$ & $34 \pm 5$ & ns \\
\hline Current/ex smokers & $474(47)$ & $251(63)$ & $<0.001$ \\
\hline Blood systolic pressure $(\mathrm{mmHg})$ & $124 \pm 15$ & $130 \pm 15$ & $<0.001$ \\
\hline Blood diastolic pressure $(\mathrm{mmHg})$ & $78 \pm 9$ & $82 \pm 10$ & $<0.001$ \\
\hline Tot-cholesterol (mg/dL) & $216 \pm 39$ & $208 \pm 41$ & $<0.001$ \\
\hline Triglycerides (mg/dL) & $110 \pm 67$ & $140 \pm 90$ & $<0.001$ \\
\hline High density lipoproteins $(\mathrm{mg} / \mathrm{dL})$ & $63 \pm 15$ & $49 \pm 11$ & $<0.001$ \\
\hline Low density lipoproteins (mg/dL) & $136 \pm 35$ & $134 \pm 35$ & ns \\
\hline Fasting glucose $(\mathrm{mg} / \mathrm{dL})$ & $92 \pm 10$ & $96 \pm 10$ & $<0.001$ \\
\hline Fasting insulin (mU/L) & $14 \pm 9$ & $17 \pm 14$ & $<0.001$ \\
\hline Creatinine $(\mathrm{mg} / \mathrm{dL})$ & $0.7 \pm 0.1$ & $1 \pm 0.5$ & $<0.001$ \\
\hline Homocysteine ( $\mu \mathrm{mol} / \mathrm{L})$ & $11 \pm 4$ & $14 \pm 7$ & $<0.001$ \\
\hline C reactive protein $(\mathrm{mg} / \mathrm{dL})$ & $0.5 \pm 0.7$ & $0.3 \pm 0.5$ & $<0.001$ \\
\hline Fibrinogen $(\mathrm{mg} / \mathrm{dL})$ & $336 \pm 62$ & $315 \pm 60$ & $<0.001$ \\
\hline Gamma glutamyltransferase (UI/L) & $22 \pm 18$ & $34 \pm 25$ & $<0.001$ \\
\hline White blood cells $\left(10^{9} / \mathrm{L}\right)$ & $0.5 \pm 0.2$ & $0.6 \pm 0.2$ & $<0.001$ \\
\hline Uric acid $(\mathrm{mg} / \mathrm{dL})$ & $4.7 \pm 1.1$ & $6.2 \pm 1.3$ & $<0.001$ \\
\hline
\end{tabular}

In Table 2 were reported the calculated IR and cardiometabolic risk indices, all higher in men, except for the QUICKI. 
Table 2

Indices of cardiometabolic risk in the overweight/obese workers according to gender

\begin{tabular}{|c|c|c|c|}
\hline & FEMALES & MALES & $\mathrm{p}$ value \\
\hline HOMA-IR & $3.2 \pm 2.4$ & $4.1 \pm 4$ & $<0.001$ \\
\hline HOMA-B (\%) & $178 \pm 116$ & $200 \pm 174$ & $<0.01$ \\
\hline QUICKI & $0.51 \pm 0.25$ & $0.46 \pm 0.13$ & $<0.001$ \\
\hline InsuTAG & $19 \pm 23$ & $29 \pm 42$ & $<0.001$ \\
\hline TYG & $8.4 \pm 0.5$ & $8.7 \pm 0.5$ & $<0.001$ \\
\hline Ty/HDLC & $2 \pm 1.8$ & $3.2 \pm 2.9$ & $<0.001$ \\
\hline TC/HDLC & $3.6 \pm 1.0$ & $4.4 \pm 1.2$ & $<0.001$ \\
\hline non-HDLC & $154 \pm 39$ & $158 \pm 40$ & ns \\
\hline $\mathrm{Al}(\mathrm{LDCL} / \mathrm{HDLC})$ & $73 \pm 39$ & $85 \pm 36$ & $<0.001$ \\
\hline TRL-C (nonHDLC-LDLC) & $18 \pm 13$ & $24 \pm 17$ & $<0.001$ \\
\hline AIP (LOG(Ty/HDLC)) & $0.2 \pm 0.26$ & $0.4 \pm 0.28$ & $<0.001$ \\
\hline VAl & $1.6 \pm 1.5$ & $1.9 \pm 1.8$ & $<0.01$ \\
\hline
\end{tabular}

Moreover Table 3 reported the correlation between the different indirect cardiometabolic indices and CV risk factors according to gender. CV risk factors more frequently correlated with cardiometabolic indices in women. Interestingly, homocysteine was more commonly associated with cardiometabolic indices in men. 
Table 3

Correlation between cardiometabolic indices and risk factors in overweight/obese workers according to gender. Background gray for parameter significativ

\begin{tabular}{|c|c|c|c|c|c|c|c|c|c|c|c|}
\hline FEMALES & $\begin{array}{l}\text { HOMA- } \\
\text { IR }\end{array}$ & $\begin{array}{l}\text { HOMA- } \\
\text { B (\%) }\end{array}$ & QUICKI & InsuTAG & TYG & Ty/HDLC & TC/HDLC & nonHDLC & $\begin{array}{l}\text { Al } \\
\text { (LDLC/HDLC) }\end{array}$ & $\begin{array}{l}\text { TRL-C } \\
\text { (nonHDLC- } \\
\text { LDLC) }\end{array}$ & $\begin{array}{l}\text { AIP } \\
\text { (LOG(Ty, }\end{array}$ \\
\hline AGE (yrs) & ns & $\begin{array}{l}r=-0.24 \\
<0.001\end{array}$ & $\begin{array}{l}r=-0.07 \\
<0.05\end{array}$ & ns & $\begin{array}{l}r=0.25 \\
<0.001\end{array}$ & $\begin{array}{l}r=0.09 \\
<0.05\end{array}$ & $\begin{array}{l}r=0.14 \\
<0.001\end{array}$ & $\begin{array}{l}r=0.3 \\
<0.001\end{array}$ & $\begin{array}{l}r=0.23 \\
<0.001\end{array}$ & $\begin{array}{l}r=0.11 \\
<0.01\end{array}$ & $r=0.12<1$ \\
\hline $\begin{array}{l}\text { Weist circunference } \\
(\mathrm{mm})\end{array}$ & $\begin{array}{l}r=0.4 \\
<0.001\end{array}$ & $\begin{array}{l}r=0.3 \\
<0.001\end{array}$ & $\begin{array}{l}r=-0.27 \\
<0.001\end{array}$ & $\begin{array}{l}r=0.34 \\
<0.001\end{array}$ & $\begin{array}{l}r=-0.35 \\
<0.001\end{array}$ & $\begin{array}{l}r=0.25 \\
<0.001\end{array}$ & $\begin{array}{l}r=0.28 \\
<0.001\end{array}$ & $\begin{array}{l}r=0.14 \\
<0.001\end{array}$ & $r=0.2<0.001$ & $\begin{array}{l}r=-0.14 \\
<0.001\end{array}$ & $r=0.4<0$. \\
\hline $\begin{array}{l}\text { Body mass index } \\
(\mathrm{kg} / \mathrm{m} 2)\end{array}$ & $\begin{array}{l}r=0.3 \\
<0.001\end{array}$ & $\begin{array}{l}r=0.28 \\
<0.001\end{array}$ & $\begin{array}{l}r=-0.22 \\
<0.001\end{array}$ & $\begin{array}{l}r=0.26 \\
<0.001\end{array}$ & $\begin{array}{l}r=0.21 \\
<0.001\end{array}$ & $\begin{array}{l}r=0.15 \\
<0.001\end{array}$ & $\begin{array}{l}r=0.17 \\
<0.001\end{array}$ & ns & $r=0.12<0.01$ & $\begin{array}{l}r=0.08 \\
<0.05\end{array}$ & $r=0.2<0$. \\
\hline Hypertension & $34 \pm 6$ & & & & & & & & & & \\
\hline dyslipidemia & $\begin{array}{l}414 \\
(40)\end{array}$ & & & & & & & & & & \\
\hline Current/ & $\begin{array}{l}687 \\
(68)\end{array}$ & & & & & & & & & & \\
\hline
\end{tabular}

ex smoking habit

\begin{tabular}{|c|c|c|c|c|c|c|c|c|c|c|c|}
\hline $\begin{array}{l}\text { Blood systolic } \\
\text { pressure (mmHg) }\end{array}$ & $\begin{array}{l}r=0.2 \\
<0.001\end{array}$ & ns & $\begin{array}{l}r=-0.09 \\
<0.01\end{array}$ & $\begin{array}{l}r=0.16 \\
<0.001\end{array}$ & $\begin{array}{l}r=0.2 \\
<0.001\end{array}$ & $\begin{array}{l}r=0.13 \\
<0.001\end{array}$ & $\begin{array}{l}r=0.15 \\
<0.001\end{array}$ & $\begin{array}{l}r=0.11 \\
<0.01\end{array}$ & $\begin{array}{l}r=0.14 \\
<0.001\end{array}$ & $\begin{array}{l}r=0.08 \\
<0.05\end{array}$ & $r=0.15<1$ \\
\hline $\begin{array}{l}\text { Blood diastolic } \\
\text { pressure }(\mathrm{mmHg})\end{array}$ & $\begin{array}{l}r=0.16 \\
<0.001\end{array}$ & ns & $\begin{array}{l}r=-0.08 \\
<0.05\end{array}$ & $\begin{array}{l}r=0.15 \\
<0.001\end{array}$ & $\begin{array}{l}r=0.18 \\
<0.001\end{array}$ & $\begin{array}{l}r=0.13 \\
<0.001\end{array}$ & $\begin{array}{l}r=0.14 \\
<0.001\end{array}$ & $\begin{array}{l}r=0.1 \\
<0.01\end{array}$ & $r=0.11<0.01$ & & $r=0.15<1$ \\
\hline $\begin{array}{l}\text { Tot-cholesterol } \\
(\mathrm{mg} / \mathrm{dL})\end{array}$ & ns & $\begin{array}{l}r=-0.1 \\
<0.01\end{array}$ & ns & $\begin{array}{l}r=0.14 \\
<0.001\end{array}$ & & & - & - & - & - & \\
\hline $\begin{array}{l}\text { Triglycerides } \\
(\mathrm{mg} / \mathrm{dL})\end{array}$ & $\begin{array}{l}r=0.3 \\
<0.001\end{array}$ & $\begin{array}{l}r=0.2 \\
<0.001\end{array}$ & $\begin{array}{l}r=-0.16 \\
<0.001\end{array}$ & - & - & - & - & & - & - & - \\
\hline $\begin{array}{l}\text { High density } \\
\text { lipoproteins } \\
(\mathrm{mg} / \mathrm{dL})\end{array}$ & $\begin{array}{l}r=-0.3 \\
<0.001\end{array}$ & $\begin{array}{l}r=-0.24 \\
<0.001\end{array}$ & $\begin{array}{l}r=0.2 \\
<0.001\end{array}$ & $\begin{array}{l}r=-0.38 \\
<0.001\end{array}$ & & - & - & - & - & - & - \\
\hline $\begin{array}{l}\text { Low density } \\
\text { lipoproteins } \\
\text { (mg/dL) }\end{array}$ & ns & ns & ns & - & - & - & - & - & - & - & - \\
\hline $\begin{array}{l}\text { Fasting glucose } \\
(\mathrm{mg} / \mathrm{dL})\end{array}$ & - & - & - & $\begin{array}{l}r=0.3 \\
<0.001\end{array}$ & - & $\begin{array}{l}r=0.17 \\
<0.001\end{array}$ & $\begin{array}{l}r=0.2 \\
<0.001\end{array}$ & $\begin{array}{l}r=0.16 \\
<0.001\end{array}$ & $r=0.2<0.001$ & $\begin{array}{l}r=0.08 \\
<0.05\end{array}$ & $r=0.2<0$. \\
\hline $\begin{array}{l}\text { Fasting insulin } \\
(\mathrm{mU} / \mathrm{L})\end{array}$ & - & - & - & - & $\begin{array}{l}r=0.45 \\
<0.001\end{array}$ & $\begin{array}{l}r=0.34 \\
<0.001\end{array}$ & $\begin{array}{l}r=0.3 \\
<0.001\end{array}$ & $\begin{array}{l}r=0.09 \\
<0.05\end{array}$ & $\begin{array}{l}r=0.18 \\
<0.001\end{array}$ & $\begin{array}{l}r=0.16 \\
<0.001\end{array}$ & $r=0.5<0$. \\
\hline Creatinine $(\mathrm{mg} / \mathrm{dL})$ & ns & ns & ns & ns & $\begin{array}{l}r=0.11 \\
<0.01\end{array}$ & ns & & $\begin{array}{l}r=0.09 \\
<0.05\end{array}$ & & & $r=0.07<1$ \\
\hline $\begin{array}{l}\text { Homocysteine } \\
\text { (mmol/L) }\end{array}$ & ns & ns & ns & ns & $\begin{array}{l}r=0.07 \\
<0.05\end{array}$ & ns & ns & ns & ns & ns & ns \\
\hline $\begin{array}{l}\text { C reactive protein } \\
(\mathrm{mg} / \mathrm{dL})\end{array}$ & $\begin{array}{l}r=0.15 \\
<0.001\end{array}$ & $\begin{array}{l}r=0.2 \\
<0.001\end{array}$ & $\begin{array}{l}r=-0.16 \\
<0.001\end{array}$ & $\begin{array}{l}r=0.2 \\
<0.001\end{array}$ & $\begin{array}{l}r=0.2 \\
<0.001\end{array}$ & $\begin{array}{l}r=0.15 \\
<0.001\end{array}$ & $\begin{array}{l}r=0.12 \\
<0.01\end{array}$ & ns & ns & $\begin{array}{l}r=0.14 \\
<0.001\end{array}$ & $r=0.2<0$. \\
\hline Fibrinogen (mg/dL) & $\begin{array}{l}r=0.11 \\
<0.01\end{array}$ & $\begin{array}{l}r=0.1 \\
<0.05\end{array}$ & $\begin{array}{l}r=-0.07 \\
<0.05\end{array}$ & $\begin{array}{l}r=0.08 \\
<0.05\end{array}$ & $\begin{array}{l}r=0.07 \\
<0.05\end{array}$ & ns & $\begin{array}{l}r=0.1 \\
<0.01\end{array}$ & ns & $r=0.07<0.05$ & $\begin{array}{l}r=0.07 \\
<0.05\end{array}$ & $r=0.1<0$. \\
\hline $\begin{array}{l}\text { Gamma } \\
\text { glutamyltransferase } \\
\text { (UI/L) }\end{array}$ & $\begin{array}{l}r=0.3 \\
<0.001\end{array}$ & $\begin{array}{l}r=0.17 \\
<0.001\end{array}$ & $\begin{array}{l}r=-0.28 \\
<0.001\end{array}$ & $\begin{array}{l}r=0.27 \\
<0.001\end{array}$ & $\begin{array}{l}r=0.34 \\
<0.001\end{array}$ & $\begin{array}{l}r=0.2 \\
<0.001\end{array}$ & $\begin{array}{l}r=0.2 \\
<0.001\end{array}$ & $\begin{array}{l}r=0.2 \\
<0.001\end{array}$ & $\begin{array}{l}r=0.18 \\
<0.001\end{array}$ & $\begin{array}{l}r=0.16 \\
<0.001\end{array}$ & $r=0.3<0$. \\
\hline $\begin{array}{l}\text { White blood cells } \\
(109 / L)\end{array}$ & $\begin{array}{l}r=0.17 \\
<0.001\end{array}$ & $\begin{array}{l}r=0.2 \\
<0.001\end{array}$ & $\begin{array}{l}r=-0.19 \\
<0.001\end{array}$ & $\begin{array}{l}r=0.2 \\
<0.001\end{array}$ & $\begin{array}{l}r=0.21 \\
<0.001\end{array}$ & $\begin{array}{l}r=0.19 \\
<0.001\end{array}$ & $\begin{array}{l}r=0.13 \\
<0.001\end{array}$ & ns & ns & $\begin{array}{l}r=0.14 \\
<0.001\end{array}$ & $r=0.2<0$. \\
\hline Uric acid (mg/dL) & $\begin{array}{l}r=0.3 \\
<0.001\end{array}$ & $\begin{array}{l}r=0.17 \\
<0.001\end{array}$ & $\begin{array}{l}r=-0.14 \\
<0.001\end{array}$ & $\begin{array}{l}r=0.33 \\
<0.001\end{array}$ & $\begin{array}{l}r=0.33 \\
<0.001\end{array}$ & $\begin{array}{l}r=0.24 \\
<0.001\end{array}$ & $\begin{array}{l}r=0.12 \\
<0.01\end{array}$ & $\begin{array}{l}r=0.16 \\
<0.001\end{array}$ & $\begin{array}{l}r=0.16 \\
<0.001\end{array}$ & $\begin{array}{l}r=0.2 \\
<0.001\end{array}$ & $\mathrm{r}=0.2<0$ \\
\hline MALES & $\begin{array}{l}\text { HOMA- } \\
\text { IR }\end{array}$ & $\begin{array}{l}\text { HOMA- } \\
\text { B (\%) }\end{array}$ & QUICKI & InsuTAG & TYG & Ty/HDLC & TC/HDLC & nonHDLC & $\begin{array}{l}\text { Al } \\
\text { (LDLC/HDLC) }\end{array}$ & $\begin{array}{l}\text { TRL-C } \\
\text { (nonHDLC- } \\
\text { LDLC) }\end{array}$ & $\begin{array}{l}\text { AIP } \\
\text { (LOG(Ty, }\end{array}$ \\
\hline AGE (yrs) & ns & $\begin{array}{l}r=-0.11 \\
<0.05\end{array}$ & ns & ns & $\begin{array}{l}r=0.13 \\
<0.05\end{array}$ & ns & ns & ns & ns & ns & ns \\
\hline $\begin{array}{l}\text { Weist circunference } \\
(\mathrm{mm})\end{array}$ & $\begin{array}{l}r=0.3 \\
<0.001\end{array}$ & $\begin{array}{l}r=0.18 \\
<0.01\end{array}$ & $\begin{array}{l}r=-0.35 \\
<0.001\end{array}$ & $\begin{array}{l}r=0.2 \\
<0.001\end{array}$ & $\begin{array}{l}r=0.11 \\
<0.05\end{array}$ & ns & ns & ns & ns & ns & ns \\
\hline $\begin{array}{l}\text { Body mass index } \\
(\mathrm{kg} / \mathrm{m} 2)\end{array}$ & $\begin{array}{l}r=0.3 \\
<0.001\end{array}$ & $\begin{array}{l}r=0.23 \\
<0.001\end{array}$ & $\begin{array}{l}r=-0.33 \\
<0.001\end{array}$ & $\begin{array}{l}r=0.17 \\
<0.01\end{array}$ & ns & ns & ns & ns & ns & ns & ns \\
\hline
\end{tabular}




\begin{tabular}{|c|c|c|c|c|c|c|c|c|c|c|c|}
\hline FEMALES & $\begin{array}{l}\text { HOMA- } \\
\text { IR }\end{array}$ & $\begin{array}{l}\text { HOMA- } \\
\text { B (\%) }\end{array}$ & QUICKI & InsuTAG & TYG & Ty/HDLC & TC/HDLC & nonHDLC & $\begin{array}{l}\text { Al } \\
\text { (LDLC/HDLC) }\end{array}$ & $\begin{array}{l}\text { TRL-C } \\
\text { (nonHDLC- } \\
\text { LDLC) }\end{array}$ & $\begin{array}{l}\text { AlP } \\
\text { (LOG(Ty, }\end{array}$ \\
\hline No smokers & 318(32) & & & & & & & & & & \\
\hline Current/ex smokers & 156(15) & & & & & & & & & & \\
\hline $\begin{array}{l}\text { Blood systolic } \\
\text { pressure }(\mathrm{mmHg})\end{array}$ & $\begin{array}{l}r=0.15 \\
<0.01\end{array}$ & ns & $\begin{array}{l}r=-0.19 \\
<0.001\end{array}$ & ns & ns & ns & ns & ns & ns & ns & \\
\hline \multirow[t]{2}{*}{$\begin{array}{l}\text { Blood diastolic } \\
\text { pressure }(\mathrm{mmHg})\end{array}$} & $\begin{array}{l}r=0.12 \\
<0.05\end{array}$ & ns & $\begin{array}{l}r=-0.17 \\
<0.01\end{array}$ & $\begin{array}{l}r=0.15 \\
<0.01\end{array}$ & ns & $\begin{array}{l}r=0.11 \\
<0.05\end{array}$ & ns & ns & ns & ns & ns \\
\hline & & & & & & & & & & & ns \\
\hline $\begin{array}{l}\text { Tot-cholesterol } \\
(\mathrm{mg} / \mathrm{dL})\end{array}$ & ns & ns & ns & $\begin{array}{l}r=0.13 \\
<0.05\end{array}$ & & & - & - & & - & \\
\hline $\begin{array}{l}\text { Triglycerides } \\
(\mathrm{mg} / \mathrm{dL})\end{array}$ & $\begin{array}{l}r=0.15 \\
<0.01\end{array}$ & $\begin{array}{l}r=0.22 \\
<0.001\end{array}$ & $\begin{array}{l}r=-0.24 \\
<0.001\end{array}$ & - & - & - & & & & - & - \\
\hline $\begin{array}{l}\text { High density } \\
\text { lipoproteins } \\
\text { (mg/dL) }\end{array}$ & $\begin{array}{l}r=-0.15 \\
<0.01\end{array}$ & $\begin{array}{l}r=-0.2 \\
<0.001\end{array}$ & $\begin{array}{l}r=0.23 \\
<0.001\end{array}$ & $\begin{array}{l}r=-0.36 \\
<0.001\end{array}$ & & - & - & - & - & - & - \\
\hline $\begin{array}{l}\text { Low density } \\
\text { lipoproteins } \\
\text { (mg/dL) }\end{array}$ & ns & ns & ns & - & - & - & - & - & - & - & - \\
\hline $\begin{array}{l}\text { Fasting glucose } \\
(\mathrm{mg} / \mathrm{dL})\end{array}$ & - & - & - & ns & - & ns & ns & ns & ns & ns & ns \\
\hline $\begin{array}{l}\text { Fasting insulin } \\
(\mathrm{mU} / \mathrm{L})\end{array}$ & - & - & - & - & $\begin{array}{l}r=0.34 \\
<0.001\end{array}$ & $\begin{array}{l}r=0.3 \\
<0.001\end{array}$ & $\begin{array}{l}r=0.2 \\
<0.001\end{array}$ & ns & ns & $\begin{array}{l}r=0.2 \\
<0.001\end{array}$ & $r=0.3<0$. \\
\hline Creatinine $(\mathrm{mg} / \mathrm{dL})$ & ns & ns & ns & ns & $\begin{array}{l}r=0.12 \\
<0.05\end{array}$ & ns & & ns & & & $r=0.12<($ \\
\hline $\begin{array}{l}\text { Homocysteine } \\
(\mathrm{mmol} / \mathrm{L})\end{array}$ & ns & ns & $\begin{array}{l}r=-0.11 \\
<0.05\end{array}$ & ns & $\begin{array}{l}r=0.2 \\
<0.001\end{array}$ & $\begin{array}{l}r=0.12 \\
<0.05\end{array}$ & $\begin{array}{l}r=0.14 \\
<0.05\end{array}$ & $\begin{array}{l}r=0.11 \\
<0.05\end{array}$ & ns & ns & $r=0.18<1$ \\
\hline $\begin{array}{l}\text { C reactive protein } \\
(\mathrm{mg} / \mathrm{dL})\end{array}$ & $\begin{array}{l}r=0.3 \\
<0.001\end{array}$ & $\begin{array}{l}r=0.18 \\
<0.01\end{array}$ & $\begin{array}{l}r=-0.19 \\
<0.001\end{array}$ & $\begin{array}{l}r=0.14 \\
<0.05\end{array}$ & ns & ns & ns & ns & ns & $\begin{array}{l}r=0.12 \\
<0.05\end{array}$ & ns \\
\hline Fibrinogen (mg/dL) & $\begin{array}{l}r=0.16 \\
<0.01\end{array}$ & $\begin{array}{l}r=0.11 \\
<0.05\end{array}$ & $\begin{array}{l}r=-0.15 \\
<0.01\end{array}$ & $\begin{array}{l}r=0.12 \\
<0.05\end{array}$ & ns & ns & $\begin{array}{l}r=0.13 \\
<0.05\end{array}$ & $\begin{array}{l}r=0.14 \\
<0.05\end{array}$ & ns & $\begin{array}{l}r=0.12 \\
<0.05\end{array}$ & ns \\
\hline $\begin{array}{l}\text { Gamma } \\
\text { glutamyltransferase } \\
\text { (UI/L) }\end{array}$ & $\begin{array}{l}r=0.16 \\
<0.01\end{array}$ & $\begin{array}{l}r=0.11 \\
<0.05\end{array}$ & $\begin{array}{l}r=-0.26 \\
<0.001\end{array}$ & $\begin{array}{l}r=0.23 \\
<0.001\end{array}$ & $\begin{array}{l}r=0.32 \\
<0.001\end{array}$ & $\begin{array}{l}r=0.25 \\
<0.001\end{array}$ & $\begin{array}{l}r=0.2 \\
<0.001\end{array}$ & $\begin{array}{l}r=0.12 \\
<0.05\end{array}$ & ns & $\begin{array}{l}r=0.22 \\
<0.001\end{array}$ & $r=0.3<0$. \\
\hline $\begin{array}{l}\text { White blood cells } \\
(109 / \mathrm{L})\end{array}$ & $\begin{array}{l}r=0.15 \\
<0.01\end{array}$ & $\begin{array}{l}r=0.17 \\
<0.01\end{array}$ & $\begin{array}{l}r=-0.15 \\
<0.01\end{array}$ & $\begin{array}{l}r=0.2 \\
<0.001\end{array}$ & $\begin{array}{l}r=0.18 \\
<0.01\end{array}$ & $\begin{array}{l}r=0.16 \\
<0.01\end{array}$ & $\begin{array}{l}r=0.16 \\
<0.01\end{array}$ & $\begin{array}{l}r=0.11 \\
<0.05\end{array}$ & ns & $\begin{array}{l}r=0.2 \\
<0.001\end{array}$ & $r=0.2<0$. \\
\hline Uric acid (mg/dL) & $\begin{array}{l}r=0.12 \\
<0.05\end{array}$ & ns & $\begin{array}{l}r=-0.3 \\
<0.001\end{array}$ & $\begin{array}{l}r=0.2 \\
<0.001\end{array}$ & $\begin{array}{l}r=0.28 \\
<0.001\end{array}$ & $\begin{array}{l}r=0.2 \\
<0.001\end{array}$ & $\begin{array}{l}r=0.16 \\
<0.01\end{array}$ & ns & ns & $\begin{array}{l}r=0.2 \\
<0.001\end{array}$ & $r=0.2<0$. \\
\hline
\end{tabular}

In Fig. 1 is reported the categorization of the IR/cardiometabolic risk status through the test utilized according to gender. It is noteworthy the variability of the percent of the workers identified as insulin resistant, IR+, or at higher cardiometabolic risk greatly vary according to the different index used.

\section{Discussion}

The present study mostly focused on a large sample of working-age subjects with overweight and obesity, where alterations in cardiometabolic indexes could be indicative of their further worsening toward overt T2DM and cardiovascular risk.

The main findings of the present study are:

1) The prevalence of IR and cardiometabolic risk was higher among males for all indices;

2) CV risk factors were correlated more frequently with IR/cardiometabolic indexes in women, especially when considering aging, waist circumference, BMI, blood pressure, glucose, CRP, fibrinogen and uric acid. Instead, homocysteine was more commonly associated with IR/cardiometabolic indexes in men;

3) The worker percentage of IR+ and subjects at higher cardiometabolic risk greatly varies according to the different index used to measure risk.

Isolated impaired fasting glucose (IFG) is generally found more prevalent in men, whereas impaired glucose tolerance, likely more related to cardiovascular risk, is more common in women [30;31]. Accordingly, we found a higher percentage of women with altered 0GTT (19\% versus $13 \%$, data not shown), which was performed in a subgroup of subjects (316 women and 106 men). However, as insulin resistance is linked closely to IFG, it is not surprising to find IR 
indices more elevated in men. Women remain more insulin sensitive, also despite weight gain, thanks to their capacity to expand subcutaneous fat, as we also observed in our population (higher QUICKI levels in women)[32]. It is known that at a given BMI the risk to develop T2D is higher in men than in women, presumably because of male higher insulin resistance [33;34]. This means that women have to increase more weight, and more adipose tissue, to develop insulin resistance and subsequently T2D. Moreover, fat distribution and quality differ by sex and, in general, men have greater visceral and hepatic fat compared with women [35]. In men ectopic fat accumulates in different organs, including the liver where it determines an increase of hepatic enzymes and triglycerides, rendering organs insulin-resistant [35]. Mechanisms determining increase of toxic lipid derivatives (ceramides or diglycerides) or other molecules are involved in this process, which, in turn, alters insulin pathway[35]. Moreover, men generally together with higher fasting glucose levels, had lower HDLC levels than women, as confirmed in our population[35].

Obesity, insulin resistance and insulin hypersecretion are likely to be key mediators of development of T2D in men. However, excess weight in women may be associated with a greater deterioration in the cardiometabolic risk. In fact, waist circumference and BMI resulted more frequently correlated with $\mathrm{IR} /$ cardiometabolic indexes in women. Moreover, the correlation more frequent between aging and IR/cardiometabolic indexes in women evidences the importance of menopause (sex hormone-e.g., oestrogen-suddenly falling with a relative growth in male hormones-e.g., testosterone). Testosterone increase in women is related to insulin resistance, hyperglycemia, increased prevalence of central obesity and hypertension. Previous evidence suggested that at the stage of prediabetes especially women have a higher risk of cardiovascular disease[36]. Biological causes related to this risk pattern involve a loss of the protective female sex hormones and their imbalance in hyperglycemic conditions leading to higher oxidative stress and inflammation, endothelial dysfunction, hypertension[37]. Accordingly, we found a more frequent correlation of CRP and uric acid (biomarkers related to inflammation and oxidative stress) with cardiometabolic indexes in women. Also the more frequent relationship between fibrinogen and cardiometabolic indexes in women may evidence an higher $\mathrm{CV}$ risk, as fibrinogen contributes to increased blood viscosity, fibrin formation and platelet aggregation[38]. Interestingly, Hcy was more commonly associated with IR/cardiometabolic indexes in men. According to previous data, Hcy was found higher in male than in females, and increases with aging and creatinine levels [39;40]. Different determinants affect Hcy levels such as diet, smoking habit, genetic and environmental factors, and hyperhomocysteinemia might be an additional risk factor in abdominal obesity[40]. Accordingly, we observed a significant relationship between VAl and homocysteine only in men ( $r=0.13$, $p<0.05$, data not shown).

For the discrepancy observed in the identification of IR+ subjects between different IR indexes, it is important to remember that these indexes have different derivation. IR has genetic and environmental components and different pathogenetic origins (e.g. mitochondrial, inflammatory, metabolic, and adipocytic)s41-45. Moreover, the indices included in their determination different biomarkers that reflect different pathophysiological pathways. For example, the Ty/HDLC has been conceived as a marker of cardiovascular risk and, as such, suitable to better identification of subjects with altered lipid profile within those affected by IR, whereas TyG, based on glucose and triglycerides values, provides information about subjects that have high risk to develop diabetes and dyslipidemia. However, the main advantage of the TyG and Ty/HDLC is that they derive from the fasting state measurements of easily available laboratory parameters, without requiring the more expensive quantification of serum insulin.

From a statistical point of view, the use of the IR indexes is, often, not accompanied by adequate information about their calculation, which may account for the discrepancy in the definition of IR-/IR+ subjects. According to literature, no explanation was reported about the selected coefficients giving an idea of the limitations of this comparison. In fact, it is difficult to establish if the coefficient is a constant, derived by others measurement, or is a parameter. In fact, a parameter, derived for example by a mean or median, should be always reported with its standard deviation, but in many cases it is difficult to find out the original formula and the consequent errors might invalidate the result of the following analyses. For example, the parameter 22.5 of HOMAIR formula represents the fasting plasma glucose/ $C$ peptide levels ratio; but it was calculated only on six healthy subjects and six diabetic patients[5] without the possibility to compute its standard deviation; therefore, it is probably not so correct to extend to a whole population a parameter calculated in a very small sample. The same problems were found for ISI 0,120 and TyG. There is no information regarding multiplying body weight by 0.19 or dividing the triglycerides and glucose product by 2 before converting it into logarithmic form. QUICKI has no parameters or constants, but its validation can be disputable, because the population sample used by Katz was small (out of 56 subjects, only 13 subjects were affected by obesity) and, moreover, all the subjects were mostly Caucasian[6].

Importantly, it can also be observed that reference intervals, decision values and cut-offs for IR and cardiometabolic risk indexes are determined in various ways. For example, majority of published studies report information about indirect insulin resistance indexes values, given as mean or median[40]. When used, cut-off for the identification of abnormalities are established as upper 75th or 95th, or below 25th or 5th percentile values observed in different examined cohorts, and applied not only to identify IR+ subjects, but often to discriminate groups of individuals with an increased risk of metabolic disorders associated with IR[46]. However, these thresholds may be different when considering different ages, gender, patient populations and ethnic groups, but also dependent on the laboratory methods used to determine biomarker concentration[46].

\section{Conclusions}

Men appear more IR than females, also presenting higher levels in cardiometabolic risk indexes. In women the IR/cardiometabolic indexes were more frequently correlated with inflammatory/oxidative stress-related biomarkers (CRP and uric acid), fasting glucose, fibrinogen, hypertension and WC and BMI. Instead, homocysteine was correlated more recurrently with IR/cardiometabolic indexes. These gender-related differences in the relationship between $\mathrm{CV}$ risk factors and IR/cardiometabolic indexes could be a potential pathophysiological determinant for the sex-related epidemiological differences in cardiometabolic diseases. 
Thus, with a small group of biomarkers and anthropometric measures it is possible to calculate a number of IR/cardiometabolic indexes, which interpretation may assist for a personalized evaluation of IR and cardiometabolic risk (Figure 1). It is true that different indexes may measure different physiopathological aspects of dysglycemia and cardiometabolic risk, giving different information, which however may also represent an advantage, being more revelatory than one single parameter reflecting only a level of the whole phenomenon.

The major advantage for the use of a panel of indices is that it requires only few biomarkers easily available and normally tested in the general patient evaluation (anthropometric measurements or simple laboratory parameters such as glucose and lipids; only insulin a little more expensive and less diffuse). In any case, it avoids the high costs and difficulties for example associated with the euglycemic-hyperglycemic clamp technique for IR+ identification, difficult to apply in the clinical practice.

\section{Declarations}

Author Contributions: VL, VC, TAS,: study conceptualization, DPS, TL, TAS, BF, TS, VL, MG data acquisition, and database assessment, CV, GM: data analysis, $\mathrm{CV}, \mathrm{VL}$ drafting of the manuscript. VL, VC, CK, BF, GM, MG, TS, TL DPS, TAS:contributed to the manuscript intellectual content and gave approval to the final version.

Conflicts of Interest: The authors declare no conflict of interest.

Funding: This research received no external funding

Institutional Review Board Statement: The study was conducted according to the Good Clinical Practice guidelines and approved by Human Ethic Committee of Fondazione IRCCS Ca' Granda Ospedale Maggiore Policlinico (Registration number: 852).

Acknowledgments: Center of Obesity and Work, Occupational Health Unit of Clinica del Lavoro "L. Devoto", Fondazione IRCCS Ca' Granda, Ospedale Maggiore Policlinico, Milan (Italy).

\section{References}

1. R.H. Eckel, S.E. Kahn, E. Ferrannini, A.B. Goldfine, D.M. Nathan, M.W. Schwartz, R.J. Smith, S.R. Smith Obesity and type 2 diabetes: What Can be unified and what needs to be individualized? In Proceedings of the Diabetes Care; 2011; Vol. 34

2. J.P. Moriarty, M.E. Branda, K.D. Olsen, N.D. Shah, B.J. Borah, A.E. Wagie, J.S. Egginton, J.M. Naessens The effects of incremental costs of smoking and obesity on health care costs among adults: A 7-year longitudinal study. J. Occup. Environ. Med. 2012, 54, doi:10.1097/JOM.0b013e318246f1f4

3. M.S. Greenfield, L. Doberne, F. Kraemer, T. Tobey, G. Reaven Assessment of insulin resistance with the insulin suppression test and the euglycemic clamp. Diabetes 1981, 30, doi:10.2337/diab.30.5.387

4. R.A. DeFronzo, J.D. Tobin, R. Andres Glucose clamp technique: A method for quantifying insulin secretion and resistance. Am. J. Physiol. Endocrinol. Metab. Gastrointest. Physiol. 1979, 6, doi:10.1152/ajpendo.1979.237.3.e214

5. M.D. R, H.J. P, R.A. S, N.B. A, T.D. F, T.R. C. Homeostasis model assessment: insulin resistance and beta-cell function from fasting plasma glucose and insulin concentrations in man. Diabetologia 1985, 28

6. A. Katz, S.S. Nambi, K. Mather, A.D. Baron, D.A. Follmann, G. Sullivan, M.J. Quon Quantitative insulin sensitivity check index: A simple, accurate method for assessing insulin sensitivity in humans. J. Clin. Endocrinol. Metab. 2000, 85, doi:10.1210/jcem.85.7.6661

7. M. Gutt, C.L. Davis, S.B. Spitzer, M.M. Llabre, M. Kumar, E.M. Czarnecki, N. Schneiderman, J.S. Skyler, J.B. Marks Validation of the insulin sensitivity index (ISI0,120): Comparison with other measures. Diabetes Res. Clin. Pract. 2000, 47, doi:10.1016/S0168-8227(99)00116-3

8. F. Tosi, E. Bonora, P. Moghetti Insulin resistance in a large cohort of women with polycystic ovary syndrome: A comparison between euglycaemichyperinsulinaemic clamp and surrogate indexes. Hum. Reprod. 2017, 32, doi:10.1093/humrep/dex308

9. T. McLaughlin, G. Reaven, F. Abbasi, C. Lamendola, M. Saad, D. Waters, J. Simon, R.M. Krauss Is there a simple way to identify insulin-resistant individuals at increased risk of cardiovascular disease? Am. J. Cardiol. 2005, 96, doi:10.1016/j.amjcard.2005.03.085

10. R. Quispe, R.J. Manalac, K.F. Faridi, M.J. Blaha, P.P. Toth, K.R. Kulkarni, K. Nasir, S.S. Virani, M. Banach, R.S. Blumenthal et al. Relationship of the triglyceride to high-density lipoprotein cholesterol (TG/HDL-C) ratio to the remainder of the lipid profile: The Very Large Database of Lipids-4 (VLDL-4) study. Atherosclerosis 2015, 242, doi:10.1016/j.atherosclerosis.2015.06.057

11. S.H. Lee, H.S. Kwon, Y.M. Park, H.S. Ha, S.H. Jeong, H.K. Yang, J.H. Lee, H.W. Yim, M. Kang, W.C. Il; Lee et al. Predicting the development of diabetes using the product of triglycerides and glucose: The chungju metabolic disease cohort (CMC) study. PLoS One 2014, 9, doi:10.1371/journal.pone.0090430

12. R.N. Thota, K.A. Abbott, J.J.A. Ferguson, M. Veysey, M. Lucock, S. Niblett, K. King, M.L. Garg InsuTAG: A novel physiologically relevant predictor for insulin resistance and metabolic syndrome. Sci. Rep. 2017, 7, doi:10.1038/s41598-017-15460-z

13. W.P. Castelli, R.D. Abbott, P.M. McNamara Summary estimates of cholesterol used to predict coronary heart disease. Circulation 1983, 67, doi:10.1161/01.CIR.67.4.730

14. X. Su, Y. Kong, D. Peng Evidence for changing lipid management strategy to focus on non-high density lipoprotein cholesterol. Lipids Health Dis. 2019,18

15. M. Dobiásová, [AIP-atherogenic index of plasma as a significant predictor of cardiovascular risk: from research to practice]. Vnitr. Lek. 2006,52

16. M.C. Amato, C. Giordano Visceral adiposity index: An indicator of adipose tissue dysfunction. Int. J. Endocrinol. 2014, 2014

17. L. Vigna, C. Vassalle, A.S. Tirelli, F. Gori, L. Tomaino, L. Sabatino, F. Bamonti Gender-related association between uric acid, homocysteine, Yglutamyltransferase, inflammatory biomarkers and metabolic syndrome in subjects affected by obesity. Biomark. Med. 2017, 11, doi:10.2217/bmm-2017-

Page $9 / 11$ 
0072

18. A. Borai, C. Livingstone, I. Kaddam, G. Ferns Selection of the appropriate method for the assessment of insulin resistance. BMC Med. Res. Methodol. 2011, 11

19. R. Muniyappa, S. Lee, H. Chen, M.J. Quon Current approaches for assessing insulin sensitivity and resistance in vivo: Advantages, limitations, and appropriate usage. Am. J. Physiol. - Endocrinol. Metab. 2008, 294

20. L.E. Simental-Mendía, M. Rodríguez-Morán, F. Guerrero-Romero The product of fasting glucose and triglycerides as surrogate for identifying insulin resistance in apparently healthy subjects. Metab. Syndr. Relat. Disord. 2008, 6, doi:10.1089/met.2008.0034

21. F. Guerrero-Romero, L.E. Simental-Mendía, M. González-Ortiz, E. Martínez-Abundis, M.G. Ramos-Zavala, S.O. Hernández-González, O. Jacques-Camarena, M. Rodríguez-Morán. The product of triglycerides and glucose, a simple measure of insulin sensitivity. Comparison with the euglycemic-hyperinsulinemic clamp. J. Clin. Endocrinol. Metab. 2010, 95, doi:10.1210/jc.2010-0288

22. F. Lucatello, L. Vigna, M. Carugno, A.S. Tirelli, P.A. Bertazzi, L. Riboldi Confronto tra indici per la valutazione dell'insulinoresistenza nella sorveglianza sanitaria dei lavoratori. G. Ital. Med. Lav. Ergon. 2012, 34

23. N. Ahn, S.E. Baumeister, U. Amann, W. Rathmann, A. Peters, C. Huth, B. Thorand, C. Meisinger Visceral adiposity index (VAl), lipid accumulation product (LAP), and product of triglycerides and glucose (TyG) to discriminate prediabetes and diabetes. Sci. Rep. 2019, 9, doi:10.1038/s41598-019-46187-8

24. J. Millán, X. Pintó, A. Muñoz, M. Zúñiga, J. Rubiés-Prat, L.F. Pallardo, L. Masana, A. Mangas, A. Hernández-Mijares, P. González-Santos et al., Lipoprotein ratios: Physiological significance and clinical usefulness in cardiovascular prevention. Vasc. Health Risk Manag<bi>.</bi> 2009, 5

25. W. Masson, M. Lobo, D. Siniawski, G. Molinero, M. Huerín, J.P. Nogueira Impact of Lipid-Lowering Therapy on Mortality According to the Baseline Non-HDL Cholesterol Level: A Meta-Analysis. High Blood Press. Cardiovasc. Prev. 2019, 26

26. A. Varbo, M. Benn, A. Tybjærg-Hansen, B.G. Nordestgaard Elevated remnant cholesterol causes both low-grade inflammation and ischemic heart disease, whereas elevated low-density lipoprotein cholesterol causes ischemic heart disease without inflammation. Circulation 2013, 128, doi:10.1161/CIRCULATIONAHA.113.003008

27. O. Castañer, X. Pintó, I. Subirana, A.J. Amor, E. Ros, Á Hernáez, M. Martínez-González, D. Corella, J. Salas-Salvadó, R. Estruch et al. Remnant Cholesterol, Not LDL Cholesterol, Is Associated With Incident Cardiovascular Disease. J. Am. Coll. Cardiol. 2020, 76, doi:10.1016/j.jacc.2020.10.008

28. M. Dobiášová, J. Frohlich The plasma parameter log (TG/HDL-C) as an atherogenic index: Correlation with lipoprotein particle size and esterification rate inapob-lipoprotein-depleted plasma (FERHDL). Clin. Biochem. 2001, 34, doi:10.1016/S0009-9120(01)00263-6

29. E. Shawaf, E. Al; Al-Ozairi, F. Al-Asfar, A. Mohammad, S. Al-Beloushi, S. Devarajan, F. Al-Mulla, J. Abubaker, H. Arefanian Atherogenic Index of Plasma (AlP) a Tool to Assess Changes in Cardiovascular Disease Risk Post Laparoscopic Sleeve Gastrectomy. J. Diabetes Res. 2020, 2020, doi:10.1155/2020/2091341

30. H. King, M. Rewers Global estimates for prevalence of diabetes mellitus and impaired glucose tolerance in adults. Diabetes Care 1993, 16, doi:10.2337/diacare.16.1.157

31. V. Regitz-Zagrosek, E. Lehmkuhl, M.O. Weickert Gender differences in the metabolic syndrome and their role for cardiovascular disease. Clin. Res. Cardiol. 2006, 95, doi:10.1007/s00392-006-0377-8

32. A.G. Huebschmann, R.R. Huxley, W.M. Kohrt, P. Zeitler, J.G. Regensteiner, J.E. ReuschB. Sex differences in the burden of type 2 diabetes and cardiovascular risk across the life course. Diabetologia 2019, 62

33. J. Logue, J.J. Walker, H.M. Colhoun, G.P. Leese, R.S. Lindsay, J.A. McKnight, A.D. Morris, D.W. Pearson, J.R. Petrie, S. Philip et al. Do men develop type 2 diabetes at lower body mass indices than women? Diabetologia 2011, 54, doi:10.1007/s00125-011-2313-3

34. S. Paul, G. Thomas, A. Majeed, K. Khunti, K. Klein Women develop type 2 diabetes at a higher body mass index than men. Diabetologia 2012,55

35. N. Sattar, Gender aspects in type 2 diabetes mellitus and cardiometabolic risk. Best Pract. Res. Clin. Endocrinol. Metab. 2013, 27

36. M.K. Breyer, A. Ofenheimer, J. Altziebler, S. Hartl, O.C. Burghuber, M. Studnicka, D. Purin, C. Heinzle, H. Drexel, F.M.E. Franssen et al. Marked differences in prediabetes- and diabetes-associated comorbidities between men and women-Epidemiological results from a general population-based cohort aged 6-80 years-The LEAD (Lung, hEart, sociAl, boDy) study. Eur. J. Clin. Invest. 2020, 50, doi:10.1111/eci.13207

37. A. Al-Salameh, P. Chanson, S. Bucher, V. Ringa, L. Becquemont Cardiovascular Disease in Type 2 Diabetes: A Review of Sex-Related Differences in Predisposition and Prevention. Mayo Clin. Proc. 2019, 94

38. V. Grossmannm, V.H. Schmitt, T. Zeller, M. Panova-Noeva, A. Schulz, D. Laubert-Reh, C. Juenger, R.B. Schnabel, T.G.J. Abt, R. Laskowski et al. Profile of the immune and inflammatory response in individuals with prediabetes and type 2 diabetes. Diabetes Care 2015, 38, doi:10.2337/dc14-3008

39. J. Dierkes, A. Jeckel, A. Ambrosch, S. Westphal, C. Luley, H. Boeing. Factors explaining the difference of total homocysteine between men and women in the European investigation into cancer and nutrition potsdam study. Metabolism. 2001, 50, doi:10.1053/meta.2001.23286

40. S. Pitla, B. Nagalla Gender-related differences in the relationship between plasma homocysteine, anthropometric and conventional biochemical coronary heart disease risk factors in middle-aged indians. Ann. Nutr. Metab. 2009, 54, doi:10.1159/000199452

41. M. D’Adamo, L. Perego, M. Cardellini, M.A. Marini, S. Frontoni, F. Andreozzi, A. Sciacqua, D. Lauro, P. Sbraccia, M. Federici et al. The -866A/A genotype in the promoter of the human uncoupling protein 2 gene is associated with insulin resistance and increased risk of type 2 diabetes. Diabetes 2004,53 , doi:10.2337/diabetes.53.7.1905

42. C. Capurso, A. Capurso, From excess adiposity to insulin resistance: The role of free fatty acids. Vascul. Pharmacol. 2012,57

43. G. Riccardi, R. Giacco, A.A. Rivellese Dietary fat, insulin sensitivity and the metabolic syndrome. Clin. Nutr. 2004, 23, doi:10.1016/j.clnu.2004.02.006

44. M.S. Zhou, I.H. Schulman, Q. Zeng, Link between the renin-angiotensin system and insulin resistance: Implications for cardiovascular disease. Vasc. Med. (United Kingdom) 2012, 17

Page $10 / 11$ 
45. H. Harizi, R. Holliday, A. Abdolahi, S.N. Georas, J.T. Brenna, X. Cai, K. Thevenet-Morrison, R.P. Phipps, P. Lawrence, S. a Mousa et al. An integrative analysis reveals coordinated reprogramming of the epigenome and the transcriptome in human skeletal muscle after training. Epigenetics 2014, 9

46. S. Placzkowska, L. Pawlik-Sobecka, I. Kokot, A. Piwowar Indirect insulin resistance detection: Current clinical trends and laboratory limitations. Biomed. Pap. 2019, 163, doi:10.5507/bp.2019.021

\section{Figures}

\section{Figure 1}

Bar charts showing the difference between the percent of at risk subjects by comparing the different indirect cardiometabolic indexes: A females, B males 